\begin{tabular}{|l|l|l||}
\hline \multicolumn{2}{|c|}{ PublisherInfo } \\
\hline \hline PublisherName & $:$ & BioMed Central \\
\hline \hline PublisherLocation & $:$ & London \\
\hline \hline PublisherImprintName & $:$ & BioMed Central \\
\hline \hline
\end{tabular}

\title{
Temporal prognostic value of ER
}

\begin{tabular}{|l|l|l||}
\hline \multicolumn{2}{|c|}{ ArticleInfo } \\
\hline \hline ArticleID & $:$ & 3734 \\
\hline \hline ArticleDOI & $:$ & $10.1186 /$ bcr-2000-66697 \\
\hline \hline ArticleCitationID & $:$ & 66697 \\
\hline \hline ArticleSequenceNumber & $:$ & 100 \\
\hline \hline ArticleCategory & $:$ & Paper Report \\
\hline \hline ArticleFirstPage & $:$ & 1 \\
\hline \hline ArticleLastPage & $:$ & 4 \\
\hline \hline & & RegistrationDate : 2000-8-25 \\
\hline ArticleHistory & $:$ & OnlineDate \\
\hline \hline ArticleCopyright & $:$ & Current Science Ltd2000-8-25 \\
\hline \hline ArticleGrants & $:$ & \\
\hline \hline ArticleContext & $:$ & 1305822 \\
\hline \hline
\end{tabular}




\section{Keywords}

Early breast cancer, ER, prognosis

\section{Introduction}

ER status (present or absent) is a well recognized prognostic and predictive factor in early breast cancer. However, there is variability in the literature as to the relative prognostic strength of ER. This variability may be due to differences in data sets, patient populations, methods of analysis, definitions and cut off points for ER, and duration of follow-up among the different studies. This study explored the impact of ER as a continuous variable in a large-node-negative breast cancer cohort with long follow-up, to re-evaluate its prognostic role.

\section{Aims}

To examine the prognostic relevance of ER and progesterone receptor $(\mathrm{PgR})$ content (as continuous variables) over time, taking into account the influence of other prognostic factors, including age, histology, and tumour size.

\section{Comments}

In this natural history study, the negative prognostic impact of increasing estrogen receptor (ER) content was found to rise over time. Thus, while the absence of ER seems to predict early recurrence, the presence of ER may predict late recurrence. These findings are thought-provoking and merit confirmation. The impact of adjuvant tamoxifen on this temporal risk is of particular interest, given that tamoxifen is now routinely given for 60 months to women with ER-positive tumors, while ER-positivity seems to become a more relevant risk factor after longer follow-up (72 months). Does tamoxifen eliminate this apparent late relationship between ER content and risk? Does it delay the emergence of this relationship? Do these findings suggest a biologic rationale for tamoxifen and anti-estrogen therapy beyond 5 years? A similar analysis in a cohort of women treated with tamoxifen might satisfactorily 
answer some of these questions. It is also important to point out that the temporal prognostic value of ER may change substantially depending on the absolute risk for the patient (using other classic prognostic factors to construct a complete prognostic profile).

\section{Methods}

The patien cohort consisted of 1793 consecutive patients from a single institution who underwent surgical excision of node-negative breast cancer with or without radiotherapy, with no systemic adjuvant therapy, and for whom at least 10 years of follow-up were available. Of these women, first relapse was locoregional in 183, distant in 225, and contralateral breast cancer occurred in $119 ; 281(15.6 \%)$ women died of breast cancer and 68 of other causes with no recurrence. ER and PgR status was evaluated using the same method for all cases. The effect of age, ER and PgR status, tumor size, and histology on disease-free survival (DFS) was examined using multiple correspondence analysis (MCA) and Cox multivariate analysis.

\section{Results}

Tumor size, histology, age, ER and PgR content were found to have independent prognostic value. ER content had no prognostic effect up to 36 months follow-up. However, after 72 months the hazard ratio was positively related to ER (ie risk increased with ER content). The impact on risk of a change in ER content was greatest at low absolute levels (1 vs $10 \mathrm{fmol} / \mathrm{mg}$ ) and less prominent at intermediate levels (10 vs $20 \mathrm{fmol} / \mathrm{mg}$ ). For age, risk decreased linearly from $<30$ to 45 years, was similar for patients 45 to 60 years, and declined linearly again with increasing age beyond 60 years. The risk of recurrence was linearly related to tumor size up to 36 months; thereafter increasing size did not adversely affect risk. Compared with ductal histology, lobular and mixed histologies had similar recurrence risk up to 80 and 70 months follow-up, respectively, and slightly higher risk thereafter.

\section{Discussion}

This paper primarily explores the impact of ER content on recurrence risk at long follow-up intervals. A linear risk for tumor size for a limited time period can be explained by early recurrence for large tumors, with a tapering off over time of the number of recurrences, thus equalizing risk for all sizes beyond a certain follow-up. Similarly, the influence of age is diminished and lost beyond early followup, which represents the high risk period. The finding that, beyond 72 months, the risk of recurrence rises linearly as ER content increases is surprising. The correlation of ER expression with adverse prognostic factors such as age and large tumor size may explain why, during early follow-up, ER does not offer independent prognostic value. With time, these factors exhaust their prognostic potential, while 
ER content becomes more relevant. Progressively greater ER levels may provide a mechanism by which latent residual malignant cells can acquire metastatic properties. In particular, estrogen inducible urokinase-type plasminogen activator and cathepsin D, two proteolytic enzymes that break down extracellular matrix, may play a role. These findings are certainly thought-provoking and merit further exploration, particularly among patients who receive adjuvant systemic therapy, which is known to modify recurrence risk.

\section{References}

1. Coradini D, Daidone MG, Boracchi P, Biganzoli E, Oriana S, Bresciani G, Pelizzaro C, Tomasic G, Di Fronzo G, Marubini E: Time-dependent relevance of steroid receptors in breast cancer. J Clin Oncol. 2000, 18: 2702-2709.

This PDF file was created after publication. 